\title{
Man and machine: Understanding the complex physiologic interactions to reduce complications
}

\author{
Nicholas G. Smedira, MD
}

Lietz and colleagues ${ }^{1}$ examined neurologic dysfunction after placement of left ventricular assist device (LVAD), an important and timely topic. Almost as important is the fact that the LVAD examined, the HeartMate XVE (Thoratec, Pleasanton, Calif), a first-generation displacement pump, will soon be of historical interest only with the successful use of continuous flow pumps. The value of this work as I saw it was to heighten our awareness of how limited our understanding of cerebral and organ perfusion during mechanical support is-whether it be too much, too little, or the wrong type-and to encourage us to intensify our investigations into understanding this in an effort to improve patient management and reduce complications.

Since the approval of the HeartMate II for bridge to transplantation in April 2008 and the use in clinical trials of third-generation centrifugal pumps such as the Duraheart (Terumo Heart, Ann Arbor, Mich), Heartware (Heartware International, Framingham, Mass), and VentrAssist (Ventracor, Foster City, Calif) pumps, continuous flow pumps have become the devices of choice at almost all major institutions, and rightfully so. Bridge-to-transplant success is greater than $70 \%$, with outstanding device reliability and durability, a threefold reduction in reoperations for bleeding, and a phenomenal 10-fold reduction in driveline infections. ${ }^{2}$ Concerns about inadequate flow in larger patients, inducing right ventricular failure and persistence of pulmonary hypertension, have been shown to be unfounded. As a result, patients and their physicians are beginning to consider mechanical circulatory support with these pumps as a worthwhile alternative for the treatment of heart failure.

What has emerged as new, vexing complications, too frequent and devastating to ignore, are gastrointestinal (GI) and intracerebral bleeding (ICB). Crow and colleagues ${ }^{3}$ have reported that GI bleeding is 10 times more frequent in patients supported with a variety of continuous flow pumps when compared with pulsatile pumps, and this rate of bleeding is much higher than seen in patients with mechanical valves and similar levels of anticoagulation. Overall, neurologic events were low in the HeartMate II trial (with the exception of women, for whom the stroke rate was $18 \%$ ), but $3 \%$ of

\footnotetext{
From the Department of Thoracic/Cardiovascular Surgery, The Cleveland Clinic, Cleveland, Ohio.

Received for publication March 9, 2009; accepted for publication March 29, 2009. Address for reprints: Nicholas G. Smedira, MD, The Cleveland Clinic Foundation, Department of Thoracic/Cardiovascular Surgery, 9500 Euclid Avenue, Desk F25, Cleveland, OH 44195 (E-mail: smedirn@ccf.org).

J Thorac Cardiovasc Surg 2009;138:9-10

$0022-5223 / \$ 36.00$

Copyright (c) 2009 by The American Association for Thoracic Surgery doi:10.1016/j.jtcvs.2009.03.048
}

patients had ICB, and in our past 50 cases, 5 patients had ICB (1 traumatic). ICB has been reported with all continuous flow pumps. ${ }^{4,5}$ Why is this?

Surprisingly, there are very few data on the cerebral blood flow response to long-term nonpulsatile flow. ${ }^{6}$ The unsubstantiated hypothesis purposed to explain GI bleeding is that similar to reduced pulsatility aortic stenosis, arterial-venous malformations form or are unmasked, leading to bleeding, especially in the setting of dual-agent anticoagulation. I could not find evidence to support this occurrence in the brain. Another theory is that the high sheer stress of continuous flow pumps results in an acquired von Willebrand factor deficiency, and this along with anticoagulation increases the risk of hemorrhagic complications. ${ }^{7,8}$ Small series have been inconsistent, and it is not clear how important this is. Device anticoagulation regimens have been modeled on therapy for prosthetic valve replacement, which may or may not be appropriate or even necessary, and the tests used to monitor levels of anticoagulation have been relatively crude and inexact. We are just beginning to understand how the patient's illness, immune response (heparininduced thrombocytopenia, for example), and mechanical properties of the pump alter the hemostatic milieu and what tests (platelet aggregation assays, thromboelastography, factor levels, and von Willebrand assays) are most useful in directing therapeutic interventions. We have also not considered that the requirement for and level of anticoagulation may change over time.

Hypertension is a risk factor for ICB. We are now discussing how to define normal blood pressure when there is little or no pulsatility. What does a radial artery Doppler-derived sound at $100 \mathrm{~mm} \mathrm{Hg}$ cuff pressure mean? It is not a mean blood pressure, as there is no diastole, and diastole or a declining blood pressure occupies two-thirds of the cardiac cycle. This would suggest that a continuous, never-varying blood pressure of 90 to 100 or more is extremely high. Lietz and colleagues ${ }^{1}$ have pointed out that baseline cerebral blood flow is reduced up to $30 \%$ in patients with advanced heart failure, and our therapeutic management often reduces systemic blood pressure to enhance cardiac output. Could normalizing blood pressure after support from a low baseline during heart failure increase the risk of ICB?

It is important to note that the patients of Lietz and colleagues ${ }^{1}$ did not have evidence of ICB, and that has been our experience with pulsatile pumps-even with the Novacor LVAD (World Heart, Oakland, Calif), which initially had extremely high stroke rates with intensive anticoagulation. Hyperperfusion neurologic dysfunction was correlated 
with higher flows but not hypertension. What flow rates, what level of blood pressure, and what degree of pulsatility, if any, are optimal for organ perfusion remain unknown.

Over the past decade, improvements in patient management and selection, and most importantly device design, have made mechanical support for both bridge and permanent therapy a safe, reliable, and realistic option for many more patients with heart failure. Improving our understanding of device-patient interaction will go a long way toward reducing complications and improving the patient's quality of life. Lietz and colleagues' work ${ }^{1}$ may already be outdated, but the idea of understanding the complex interplay of device flow characteristics and organ physiology is more important than ever.

\section{References}

1. Lietz K, Brown K, Ali SS, et al. The role of cerebral hyperperfusion in postoperative neurologic dysfunction after left ventricular assist device im- plantation for end-stage heart failure. $J$ Thorac Cardiovasc Surg. 2009;137: 1012-9.

2. Miller LW, Pagani FD, Russell SD, et al. Use of a continuous-flow device in patients awaiting heart transplantation. $N$ Engl J Med. 2008;357: 886-96.

3. Crow S, John R, Boyle A, et al. Gastrointestinal bleeding rates in recipients of nonpulsatile and pulsatile left ventricular assist devices. J Thorac Cardiovasc Surg. 2009;137:208-15.

4. Esmore D, Kaye D, Spratt P, et al. A prospective, multicenter trial of the VentrAssist $^{\mathrm{TM}}$ left ventricular assist device for bridge to transplant: safety and efficacy. J Heart Lung Transplant. 2008;27:579-88.

5. Nojiri C, Fey O, Jaschke F, et al. Long-term circulatory support with the DuraHeart ${ }^{\mathrm{TM}}$ Mag-Lev Centrifugal left ventricular assist system for advanced heart failure patients eligible to transplantation: European experiences. J Heart Lung Transplant. 2008;27:S245.

6. Thalmann M, Schima H, Wieselthaler G, et al. Physiology of continuous blood flow in recipients of rotary cardiac assist devices. J Heart Lung Transplant. 2005;24:237-45.

7. Steinlechner B, Dworschak M, Birkenberg B, et al. Platelet dysfunction in outpatients with left ventricular assist devices. Ann Thorac Surg. 2009;87: 131-8.

8. Geisen U, Heilmann C, Beyersdorf F, et al. Non-surgical bleeding in patients with ventricular assist devices could be explained by acquired von Willebrand disease. Eur J Cardiothorac Surg. 2008;33:679-84. 